\title{
RINGS WHICH RESEMBLE RINGS OF ENTIRE FUNCTIONS
}

\author{
by DAVID E. RUSH
}

(Received 16 March, 1981)

Since Helmer's 1940 paper [9] laid the foundations for the study of the ideal theory of the ring $A(\mathbb{C})$ of entire functionst, many interesting results have been obtained for the rings $A(X)$ of analytic functions on non-compact connected Riemann surfaces. For example, the partially ordered set $\operatorname{Spec}(A(\mathbb{C}))$ of prime ideals of $A(\mathbb{C})$ has been described by Henrikson and others [2], [10], [11]. Also, it has been shown by Alling [4] that $\operatorname{Spec}(A(\mathbb{C})) \cong \operatorname{Spec}(A(X))$ as topological spaces for any non-compact connected Riemann surface $X$. Many results on the valuation theory of $A(X)$ have also been obtained [1], [2]. In this note we show that a large portion of the results on the rings $A(X)$ extend to the $W$-rings with complete principal divisor space which were defined by $J$. Klingen in $[15],[16]$. Therefore, many properties of $A(\mathbb{C})$ are shared by its non-archimedian counterparts studied by M. Lazard, M. Krasner, and others [8], [17], [18].

In $\$ 1$ we give the relevant definitions and then give some conditions on a $W$-ring $R$ which are equivalent to the condition that $R$ satisfy a Mittag-Leffler theorem, and also give some applications. In $\$ 2$ we consider the group of divisibility and indicate how results of Alling [1], [2], [3], [4] on the ideal theory and valuation theory of meromorphic function fields can be extended to Klingen's more abstract setting. We conclude in $\$ 3$ with some remarks on realizing a $W$-ring as a ring of analytic functions on a Riemann surface. Since much of the work in this note involves fairly straight-forward translations to $W$-rings of known results on rings of analytic functions, the details will be kept to a minimum.

1. W-rings. We recall the definitions from [16] that we will use.

Definition 1.1. An integral domain $R$ is called a topological ZPE-domain if the following hold:

$(T) R$ is a Hausdorff topological ring in which the first countability axiom holds and all principal ideals are closed.

$(Z P 1) R$ is a GCD-domain.

$(Z P 2) R$ is topologically factorial; that is for every non-unit $x \in R$ there exists a sequence $\left\{p_{i}\right\}_{i=1}^{N}$ of pair-wise nonassociate prime elements, where $N$ is a natural number or $\infty$, a sequence $\left\{n_{i}\right\}_{i=1}^{N}$ of natural numbers, and a sequence $\left\{\epsilon_{i}\right\}_{i=1}^{N}$ of units of $R$, such that $\prod_{i=1}^{N}\left(p_{i}^{n_{i}} \epsilon_{i}\right)$ converges in $R$ to $x$. Further, the sequence $\left\{\left(p_{i} R, n_{i}\right)\right\}_{i=1}^{N}$ is unique up to order.

$\dagger$ It has been pointed out to the author by N. L. Alling that the main result of [9] can actually be found in J. H. M. Wedderburn's paper: On matrices whose coefficients are functions of a single variable, Trans. Amer. Math. Soc. 16 (1915), 328-332.

Glasgow Math. J. 24 (1983) 7-16. 
If $R$ is a topological ZPE-domain we will denote the set of non-zero principal prime ideals of $R$ by $X(R)$, or just $X$ if no confusion can rise. By [16, Satz 1, p. 62] $R_{\mathrm{P}}$ is a rank one discrete valuation ring for each $P \in X$. We will denote by $v_{P}$ the associated normalized valuation, or sometimes $v_{p}$ if $P=p R$.

Definition 1.2. If $R$ is a Hausdorff topological ring, a set $\mathscr{P}$ of prime ideals of $R$ is said to be permissible if $\mathscr{P}$ is finite, or if $\mathscr{P}$ is countable and for some (equivalently, for every) numbering $\left\{P_{i}\right\}_{i=1}^{\infty}$ of $\mathscr{P}$, a sequence $\left\{r_{i}\right\}_{i=1}^{\infty}$ with $r_{i} \in P_{i}$ exists such that

$$
\lim _{i \rightarrow \infty} r_{i}=1 \text {. }
$$

We say that a set (or sequence) of prime elements $\left\{p_{i}\right\}_{i=1}^{\infty}$ is permissible if $\left\{p_{i} R\right\}_{i=1}^{\infty}$ is permissible.

If $R$ is a topological ZPE-domain and $x \in R$, then $\left\{P \in X \mid v_{P}(x) \neq 0\right\}$ is a permissible set $[16$, p. 62, Lemma 2].

Definttion 1.3. A topological ZPE-domain $R$ is said to be a $W$-ring if for every permissible sequence $\left\{p_{i}\right\}_{i=1}^{\infty}$ of prime elements of $R$, and every sequence $\left\{n_{i}\right\}_{i=1}^{\infty}$ of positive integers, there exists a sequence $\left\{\epsilon_{i}\right\}_{i=1}^{\infty}$ of units (called a convergence producing factor system for $\left.\left\{\left(p_{i}, n_{i}\right)\right\}_{i=1}^{\infty}\right)$, such that the product $\prod_{i=1}^{\infty}\left(p_{i}^{n_{i}} \epsilon_{i}\right)$ converges in $R-\{0\}$.

Several examples of topological ZPE-rings are given in [15], [16]. In particular, if a domain $R$ is a topological ZPE-domain, then so is the polynomial ring $R[X][16$, Satz 4, p. 65]. At present however, the only examples of $W$-rings known to the author are the rings $A(X)$ of analytic functions on a Riemann surface, and the non-archimedian counterparts of $A(\mathbb{C})$ which were investigated in [8], [17], [18]. These latter rings will be defined in $\$ 2$.

DefinItion 1.4. A topological ZPE-domain $R$ is said to have representation field $k$ if $k$ is a subfield of $R$ which is mapped onto $R / P$ by the canonical map for each $P \in X$.

Let $R$ be a topological ZPE-domain with quotient field $F$ and representation field $k$, and let $\mathbb{P}(R)$ be a set of representatives for the prime elements of $R$. Let $f \in F, p \in \mathbb{P}(R)$ and $v_{p}(f)=m$. Then it follows as in [16, Lemma 4] that there exist unique $a_{i} \in k$ such that $v_{p}\left(f-\sum_{i=m}^{n} a_{i} p^{i}\right)>n$ for each integer $n \geq m$. Then $\sum_{i=m}^{n} a_{i} p^{i}$ is called the $n$-th partial sum of $f$ at $p$. If $m \leq n=-1$, then $\sum_{i=m}^{n} a_{i} p^{i}$ is called the principal part of $f$ at $p$.

Definition 1.5. Let $R$ be a topological ZPE-domain with representation field $k$ and quotient field $F$ and let $\psi: F \rightarrow \prod_{P \in X} F / R_{P}$ be the canonical map. Then $\psi(F)$ is.called the principal divisor space of $R$ and is denoted $\mathrm{HT}(R)$.

In [16] $\mathrm{HT}(R)$ is given a topology so that $\mathrm{HT}(R)$ becomes a topological vector space over $k$ where $k$ is given the discrete topology. The only fact we need about the topology is 
the following. Let

$$
\prod_{P \in X}^{*} F / R_{P}=\left\{\alpha \in \prod_{P \in X} F / R_{P} \mid \text { support of } \alpha \text { is permissible }\right\} .
$$

Then $\mathrm{HT}(R) \subseteq \prod_{P \in X}^{*} F / R_{P}$ with equality exactly when $\mathrm{HT}(R)$ is complete [16, Proposition
3].

THEOREM 1.1. Let $R$ be $a W$-ring with quotient field $F$ and representation field $k$. The following properties of $R$ are equivalent.

(1) $\operatorname{HT}(R)$ is complete.

(2) $\psi: F \rightarrow \prod_{P \in X}^{*} F / R_{P}$ is surjective.

(3) For any permissible set $D \subseteq \mathbb{P}(R)$ and any set of polynomials $\left\{h_{p}\right\}_{p \in D}$ in $R[X]$ with $h_{p}(0)=0$ for all $p \in D$, there exists $f \in F$ with principal part $h_{p}\left(p^{-1}\right)$ at $p$ for $p \in D$, and $v_{p}(f) \geq 0$ for $p \in \mathbb{P}(R) \backslash D$.

(4) For any permissible set $D \subseteq \mathbb{P}(R)$ and any set $\left\{\sum_{i=m_{p}}^{n_{p}} a_{i p} p^{i}\right\}_{p \in D}$ of partial sums, there exists $f \in F$ with $n_{\mathrm{p}}$-th partial sum $\sum_{i=m_{\mathrm{p}}}^{n_{\mathrm{p}}} a_{i p} p^{i}$ at $p \in D$ and $v_{p}(f) \geq 0$ for $p \in \mathbb{P}(R) \backslash D$.

(5) For any permissible set $D \subseteq \mathbb{P}(R)$, and any family $\left\{f_{p}\right\}_{p \in D}$ of elements of $F$, and integers $\left\{n_{p}\right\}_{p \in D}$ there exists $f \in F$ such that $v_{p}\left(f-f_{p}\right)>n_{p}$ for $p \in D$ and $v_{p}(f) \geq 0$ for $p \in \mathbb{P}(R) \backslash D$. of $R$.

(6) For any $f \in R, R / f R \cong \prod_{i=1}^{\infty} R / p_{i}^{n_{i}} R$ where $f=\prod_{i=1}^{\infty}\left(p_{i}^{n_{1}} \epsilon_{i}\right)$ with $p_{i} \in \mathbb{P}(R)$ and $\epsilon_{i}$ units

Proof. (1) $\Leftrightarrow(2)$. [16, p. 71, Corollary].

$(2) \Leftrightarrow(3)$. This is immediate from the definitions.
$(3) \Rightarrow(4)$. Let $D \subseteq \mathbb{P}(R)$ be permissible and for each $p \in D$ let $A_{p}=\sum_{i=m_{p}}^{n_{p}} a_{i p} p^{i}$ be a given partial sum. Since $R$ is a $W$-ring there exists a convergence producing factor system $\left\{\epsilon_{p}\right\}_{p \in D}$ so that $g=\prod_{p \in D}\left(p^{n_{p}+1} \epsilon_{p}\right) \in R$. Let the $\left(2 n_{p}-m_{p}+1\right)$ th partial sum at $p$ of $g$ be $B_{p}$. By [1, Proposition 1.2] there exists $C_{p}=\sum_{i=0}^{n_{p}-m_{p}} c_{i p} p^{i}$ such that

$$
v_{p}\left(p^{-\left(n_{p}+1\right)} B_{p} C_{p}-p^{-m_{p}} A_{p}\right) \geq n_{p}-m_{p}+1
$$

But then $v_{p}\left(p^{\left(m_{p}-n_{p}-1\right)} B_{p} \cdot C_{p}-A_{p}\right) \geq n_{p}+1$. Since $p^{\left(m_{p}-n_{p}-1\right)} C_{p}$ is a principal part by (3) there exists $h \in F$ such that the principal part $H_{p}$ of $h$ at $p$ is $C_{p} \cdot p^{\left(m_{p}-n_{p}-1\right)}$, and $v_{p}(h) \geq 0$ for $p \notin D$.

Claim. $f=h g$ has partial sum $A_{p}$ at $p$ for each $p \in D$. 
Indeed let $h=C_{p} p^{\left(m_{p}-n_{p}-1\right)}+h^{\prime}$ and $g=B_{p}+g^{\prime}$ where $v_{p}\left(h^{\prime}\right) \geq 0$ and $v_{p}\left(g^{\prime}\right) \geq$ $2 n_{p}-m_{p}+2$. Then

$$
\begin{aligned}
v_{p} & \left(h g-A_{p}\right)=v_{p}\left[\left(C_{p} p^{\left(m_{p}-n_{p}-1\right)}+h^{\prime}\right)\left(B_{p}+g^{\prime}\right)-A_{p}\right] \\
& =v_{p}\left(C_{p} p^{\left(m_{\mathrm{p}}-n_{p}-1\right)} B_{p}+h^{\prime} B_{p}+C_{p} p^{\left(m_{p}-n_{p}-1\right)} g^{\prime}+h^{\prime} g^{\prime}-A_{p}\right) \\
& =v_{p}\left[\left(C_{p} p^{\left(m_{p}-n_{p}-1\right)} B_{p}-A_{p}\right)+h^{\prime} B_{p}+C_{p} p^{\left(m_{p}-n_{p}-1\right)} g^{\prime}+h^{\prime} g^{\prime}\right] \\
& \geq \min \left\{v_{p}\left(B_{p} C_{p} p^{\left(m_{p}-n_{p}-1\right)}-A_{p}\right), v_{p}\left(h^{\prime} B_{p}\right), v_{p}\left(C_{p} p^{\left(m_{p}-n_{p}-1\right)} g^{\prime}\right), v_{p}\left(h^{\prime} g^{\prime}\right)\right\} \\
& \geq \min \left\{n_{p}+1, n_{p}+1, m_{p}-n_{p}-1+2 n_{p}-m_{p}+2,2 n_{p}+2\right\} \\
& =n_{p}+1 .
\end{aligned}
$$

$(4) \Rightarrow(5)$. It suffices to consider the case that $n_{p} \geq v_{p}\left(f_{p}\right)$ for every $p \in D$. Let $f_{p}=$ $\left(\sum_{i}^{n_{\underline{p}}} m_{\mathrm{p}} a_{i p} p^{i}\right)+g_{p}$ where $v_{p}\left(g_{p}\right)>n_{p}$. By part (4) there exists $f \in F$ such that $f=$ $\left(\sum_{i=m_{p}}^{n} a_{i p} p^{i}\right)+h_{p}$ where $v_{p}\left(h_{p}\right)>n_{p}$ for $p \in D$ and $v_{p}(f) \geq 0$ for $p \in \mathbb{P}(R) \backslash D$. Then

$$
v_{p}\left(f-f_{p}\right)=v_{p}\left(h_{p}-g_{p}\right) \geq \min \left(v_{p}\left(h_{p}\right), v_{p}\left(g_{p}\right)\right) \geq n_{p}+1
$$

for all $p \in D$ and $v_{p}(f) \geq 0$ for $p \notin D$.

$(5) \Rightarrow(6)$. Let $\left(\bar{g}_{i}\right)_{i \in N} \in \prod_{i=1}^{\infty} R / p_{i}^{n_{i}} R, g_{i} \in R$. By (5) there exists $g \in F$ such that $v_{p_{i}}\left(g-g_{i}\right) \geq n_{i}$ and $v_{p}(g) \geq 0$ for $p \in \mathbb{P}(R) \backslash\left\{p_{i}\right\}_{i \in N}$. Then

$$
v_{p_{i}}(g)=v_{p_{i}}\left(g-g_{i}+g_{i}\right) \geq \min \left\{v_{p_{i}}\left(g-g_{i}\right), v_{p_{i}}\left(g_{i}\right)\right\} \geq 0,
$$

so $g \in R$. Further $v_{p_{i}}\left(g-g_{i}\right) \geq n_{i} \Rightarrow g \equiv g_{i}\left(\bmod p_{i}^{n_{i}} R\right)$. Thus the canonical map $R \rightarrow$ $\prod_{i=1}^{\infty} R / p_{i}^{n_{1}} R$ is surjective. Its kernel is clearly $f R$.

(6) $\Rightarrow(2)$. Let $\left\{\sum_{i=-m_{p}}^{-1} a_{i p} p^{i}\right\}_{p \in D}$ be a set of principal parts where $D \subseteq \mathbb{P}(R)$ is permissible. Let $g=\prod_{p \in D}\left(p^{m} \epsilon_{p}\right)$ for some convergence producing factor system $\left\{\epsilon_{p}\right\}_{p \in D}$. Since the canonical map $R \rightarrow \prod_{p \in D} R / p^{m_{\mathrm{p}}} R$ is surjective, there exists $f \in R$ such that $f \equiv g \sum_{i=-m_{p}}^{-1} a_{i p} p^{i}$ $\left(\bmod p^{m_{p}} R\right)$ for every $p \in D$. Thus $v_{p}\left(f-g \sum_{i=-m_{p}}^{-1} a_{i p} p^{i}\right) \geq m_{p}$ and therefore $v_{p}\left(f / g-\sum_{i=-m_{p}}^{-1} a_{i p} p^{i}\right) \geq 0$. Therefore $f / g \in F$ with principal parts $\left\{\sum_{i=-m_{p}}^{-1} a_{i p} p^{i}\right\}_{p \in D}$.

The above theorem allows us to give a very simple proof of the following result of Klingen [16, Satz 6].

THEOREM 1.2. If $R$ is a W-ring with $\mathrm{HT}(R)$ complete, then $R$ is Bezout; that is, every finitely generated ideal of $R$ is principal.

Proof. It suffices to show that if $f, g \in R$ have no common non-unit factors, then there exists $h, t \in R$ such that $h f+t g=1$. Let $g=\prod_{i \in N}\left(p_{i}^{n_{i}} \epsilon_{i}\right)$, where the $\epsilon_{i} \in R$ are units. We must find $h \in R$ such that $(1-h f) / g \in R$; that is we must find $h \in R$ such that $1-h f \equiv$ $0\left(\bmod p_{i}^{n_{i}} R\right)$ for all $i \in N$. By part $(6)$ above it suffices to show that for each $i \in N$ there 
exists $h_{i} \in R$ such that $1 \equiv h_{i} f\left(\bmod p_{i}^{n_{1}} R\right)$. But since no $p_{i}$ divides $f, i \in N, f$ is a unit $\bmod p_{i}^{n_{1}} R$ so this is clear.

Many properties of $W$-rings with complete principal divisor space can be derived via Theorem 1.1 from facts about countable products of rank one discrete valuation rings. For example the ideas in [5] yield the following:

THEOREM 1.3. Let $R$ be a non-Noetherian $W$-ring with representation field $k$ and $\mathrm{HT}(R)$ complete.

(a) If $M$ is a maximal ideal of $R, M R_{M}$ is principal.

(b) If $k$ is algebraically closed, then $R / M$ is algebraically closed for each maximal ideal $M$ of $R$.

(c) Every non-zero prime ideal of $R$ is contained in a unique maximal ideal of $R$.

(d) If $M$ is a maximal ideal of $R, Q=\bigcap_{n=1}^{\infty} M^{n}$ is the largest non-maximal prime ideal contained in $M$, and $R / Q$ is a rank one discrete valuation ring.

(e) There exists a maximal ideal $M$ of $R$ such that $Q=\bigcap_{i=1}^{\infty} M^{n} \neq\{0\}$, and for such an $M, R / Q$ is complete and $M$ contains a chain of prime ideals of length $2^{\aleph_{0}}$.

2. The group of divisibility. If $R$ is a $W$-ring, let $X_{0}$ be the set $X=X(R)$ with the topology inherited from $\operatorname{Spec}(R)$ with the Zariski topology. It follows that the closed sets of $X_{0}$ are $X$ and the permissible subsets of $X$, and that the group of divisibility $G(R)$ of $R$ is isomorphic to $\left\{\alpha \in Z^{X} \mid \operatorname{Supp}_{X}(\alpha)\right.$ is permissible $\}$ where $\operatorname{Supp}_{X}(\alpha)=\{P \in X \mid \alpha(P) \neq 0\}$. Thus $G(R)$ is completely determined by $X_{0}$. If also $R$ is Bezout (e.g., if $H T(R)$ is complete), then $G(R)$ completely determines $\operatorname{Spec}(R)$ as a partially ordered set by [7, p. 197]. In fact $G(R)$ determines $\operatorname{Spec}(R)$ as a topological space (and more) as the next theorem shows. We will use the following terminology and notation.

Definition 2.1. A proper subset $J$ of a lattice ordered abelian group $G$ is a dual ideal if the following hold:

(1) If $a, b \in J, \inf (a, b) \in J$, and

(2) if $a \in J, g \in G$, and $g \geq a$, then $g \in J$.

If $G$ is a lattice ordered abelian group let $G_{+}=\{g \in G \mid g \geq 0\}, \operatorname{di}(G)=$ the set of dual ideals of $G, J(G)=\{J \in \operatorname{di}(G) \mid$ there exists $d \in G$ such that $d \leq j$ for all $j \in J\}$, and $J\left(G_{+}\right)=$ $\left\{J \in \operatorname{di}(G) \mid J \subseteq G_{+}\right\}$. A dual ideal $J \in J\left(G_{+}\right)$is called prime (respectively primary) if $a, b \in G_{+} \backslash J$ implies $a+b \in G_{+} \backslash J$ (respectively $a, n b \in G_{+} \backslash J$ for $n=1,2, \ldots$ implies $a+b \in$ $\left.G_{+} \backslash J\right)$. For $a, b \in G$, let $a \wedge b=\inf \{a, b\}$.

THEOREM 2.1. If $R$ is a Bezout domain with quotient field $K$ and group of divisibility $G$, then the canonical map $w: K \rightarrow G \cup\{\infty\}$ gives a bijection from the set of $R$-submodules of $K$ onto the set di $(G)$, and carries the sets of fractional ideals, integral ideals, prime ideals, and primary ideals onto the sets $J(G), J\left(G_{+}\right)$, and the sets of prime and primary dual ideals, 
respectively. Further $w$ has the properties

and

(a) $w\left(I_{1}+I_{2}\right)=w\left(I_{1}\right) \wedge w\left(I_{2}\right)=\left\{j_{1} \wedge j_{2} \mid j_{1} \in w\left(I_{1}\right), j_{2} \in w\left(I_{2}\right)\right\}$,

(b) $w\left(I_{1} I_{2}\right)=w\left(I_{1}\right)+w\left(I_{2}\right)=\left\{\bigwedge_{r=1}^{n}\left(j_{1 r}+j_{2 r}\right) \mid j_{1 r} \in w\left(I_{1}\right), j_{2 r} \in w\left(I_{2}\right), r=1,2, \ldots, n\right\}$,

(c) $w\left(I_{1} \cap I_{2}\right)=w\left(I_{1}\right) \cap w\left(I_{2}\right)$

Proof. The first statement follows as in [7, p. 197]. The rest is given in [4, §2] for rings of analytic functions and easily extends to arbitrary Bezout domains. As an example we consider part $(a)$ which appears in [4] to be the least straightforward. First note that if $J, J^{\prime}$ are dual ideals of a lattice ordered abelian group $G$, then $J \wedge J^{\prime}$ is a dual ideal [4, Lemma 2.8]. Let $a \in I_{1}, b \in I_{2}$. Then since $R$ is Bezout, $a R+b R=c R$ for some $c \in R$. Then $a+b=r c$ for some $r \in R$ and so

$$
w(a+b)=w(r c)=w(r)+w(c) \geq w(c)=w(a) \wedge w(b) ;
$$

so $w\left(I_{1}+I_{2}\right) \subseteq w\left(I_{1}\right) \wedge w\left(I_{2}\right)$. Conversely, let $w(a) \wedge w(b) \in w\left(I_{1}\right) \wedge w\left(I_{2}\right), a \in I_{1}, b \in I_{2}$. Then again there exists $c \in R$ such that $c R=a R+b R$, say $c=r a+s b, r, s \in R$. Then $w(a) \wedge w(b)=w(c) \in w\left(I_{1}+I_{2}\right)$, so $w\left(I_{1}\right) \wedge w\left(I_{2}\right) \subseteq w\left(I_{1}+I_{2}\right)$.

Let $k$ be an algebraically closed field which is complete with respect to a nonarchimedian valuation ||$_{v}$. Let $L_{k}$ be the ring consisting of all Laurent series $\sum_{i=-\infty}^{\infty} a_{i} X^{i}, a_{i} \in k$ such that $\sum_{i=-\infty}^{\infty} a_{i} t^{i}$ converges for every $t \in k$. Then by $[8,17,18] L_{k}$ shares many properties of the ring $A(\mathbb{C})$ of entire functions. In $[15$, Satz 5.2] it was shown that $L_{k}$ is a $W$-ring with representation field $k$ and $\operatorname{HT}\left(L_{k}\right)$ complete. The following result shows that if $k$ has cardinality $2^{\aleph_{0}}$, then the ideal theory of $L_{k}$ is virtually identical to that of $A(X)$ for any non-compact connected Riemann surface $X$.

THEOREM 2.2. Let $k$ be an algebraically closed field which is complete with respect to a non-archimedian valuation ||$_{v}$. If $k$ and $\mathbb{C}$ have the same cardinality, then for any non-compact connected Riemann surface $X, L_{k}$ and $A(X)$ have isomorphic groups of divisibility, and therefore isomorphic lattices of ideals.

Proof. By [4, Theorem 2.3] it suffices to consider the case $X=\mathbb{C}$. Let $R=L_{k}$. From $[15$, Lemma 5.2] we get that there are canonical bijections $k \rightarrow X(R)$ and $\mathbb{C} \rightarrow X(A(\mathbb{C})$, defined by $a \rightarrow(X-a) R$ and $a \rightarrow(X-a) A(\mathbb{C})$. Let $U_{n}=\left\{\left.a \in k|| a\right|_{v}<n\right\}$ and $V_{n}=$ $\{a \in \mathbb{C}|| a \mid<n\}$ for each positive integer $n$.

Now $U_{n} \backslash U_{n-1}$ is uncountable for each $n \geq 1$ since for each $t \in k$, $\operatorname{card}\left\{\left.a \in k|| a\right|_{v}=t\right\}=\operatorname{card}\left\{\left.a \in k|| a\right|_{v}=1\right\}$. Thus for each $n$ there is a bijection $\varphi_{n}: U_{n} \backslash U_{n-1} \rightarrow V_{n} \backslash V_{n-1}$. But since $U_{n} \backslash U_{n-1}$ and $V_{n} \backslash V_{n-1}$ inherit from $X_{0}(R)$ and $X_{0}(A(\mathbb{C}))$ the cofinite topologies [15, Lemma 5.2], $\varphi_{n}$ is a homeomorphism for each $n$. The $\varphi_{n}$ patch together to give a homeomorphism $\varphi: X_{0}(R) \rightarrow X_{0}(A(\mathbb{C}))$. But as observed before, this implies $R$ and $A(\mathbb{C})$ have isomorphic groups of divisibility.

NotE. The cardinality condition in the above theorem is obviously necessary. 
As in the case of analytic functions one can obtain information about $\operatorname{Spec}(R)$ for more general $W$-rings $R$, and also information about the valuation theory of such rings, by using the correspondence between ideals of $R$ and the $\Delta$-filters of $X(R)$. We adapt the notation of [4] to our setting. If $R$ is a $W$-ring and $r \in R$, let $Z(r)=\{P \in X(R) \mid r \in P\}$. For any subset $S$ of $R$, let $Z(S)=\{Z(r) \mid r \in S\}$, and let $\Delta=Z(R)$. Then $\Delta$ is the set of Zariski closed subsets of $X(R)$.

Definition 2.2. A $\Delta$-filter on $X$ is a subset $\delta$ of $\Delta$ such that:

(a) $\varnothing \notin \delta$

(b) $U, V \in \delta \Rightarrow U \cap V \in \delta$.

(c) $U \in \delta, V \in \Delta$ and $U \subseteq V \Rightarrow V \in \delta$

A maximal $\Delta$-filter is called a $\Delta$-ultrafilter.

The following lemma is a straightforward extension of a well-known result on rings of functions $[4,10]$.

Lemma. If $I$ is a proper ideal of a $W$-ring $R$ then $Z(I)$ is a $\Delta$-filter. Conversely, if $\delta$ is a $\Delta$-filter, $Z^{-1}(\delta)$ is a proper ideal of $R$. Further $I \subseteq Z^{-1} Z(I)$, so the set of maximal ideals of $R$ is in one-to-one correspondence with the set of $\Delta$-ultrafilters on $X$.

A $\Delta$-ultrafilter $\delta$ is called fixed if $\bigcap\{D \mid D \in \delta\} \neq \varnothing$, and is called free otherwise. A maximal ideal $M$ of $R$ is called fixed (respectively free) if $Z(M)$ is fixed (respectively free). Let $R$ be a $W$-ring with $\mathrm{HT}(R)$ complete and let $M$ be a free maximal ideal of $R$. As in the case of rings $A(X)$ of analytic functions [2, p. 11] we can realize $R / M$ as an ultra-power of $k$. Indeed let $\delta=Z(M)$ and let $D \in \delta, D \neq X$. Then $\mu=\{D \cap E \mid E \in \delta\}$ is an ultrafilter on $D$, and we have a homomorphism $\varphi: R \rightarrow k^{D}$ defined by $\varphi(r)=\tilde{r} \mid D$ where $\tilde{r}: X \rightarrow k$ is defined by $\tilde{r}(p)$ is the residue class of $r$ in $R / p=k$. Let $M^{\prime}=$ $\left\{f \in k^{D} \mid f^{-1}(0) \in \mu\right\}$. Then $M=\varphi^{-1}\left(M^{\prime}\right)$ and so we have a natural injection $\bar{\varphi}: R / M \rightarrow$ $k^{D} / M^{\prime}$. Further, since $R$ is a $W$-ring with $\operatorname{HT}(R)$ complete, $\varphi$ is onto by Theorem 1 , and thus $\bar{\varphi}$ is an isomorphism. This gives another proof that $R / M$ is algebraically closed if $k$ is. Further, we find that if $k$ is an infinite field, then $M$ is principal if and only if the canonical map $k \rightarrow R / M$ is onto, and that the fixed maximal ideals of $R$ are just the elements of $X(R)$.

The value groups of the valuation rings $R_{M}$ may also be represented as ultrapowers as follows. Let $M$ be a maximal ideal of the $W$-ring $R$ having $\mathrm{HT}(R)$ complete. If $M \in X$ then clearly $G\left(R_{M}\right)=Z$. If $M$ is free then consider the canonical map $v:(K \backslash\{0\}) \rightarrow$ $G(R)=\left\{\alpha \in Z^{X} \mid \operatorname{Supp}_{X}(\alpha) \neq X\right.$ and is closed in $\left.X_{0}\right\}$. If $D \in \delta=Z(M), D \neq X$, then restriction to $D$ gives us an order preserving group homomorphism $\rho: G(R) \rightarrow Z^{D}$. Then $\rho . v$ is onto since $R$ is a $W$-ring. Let $\mu$ be the ultrafilter $\mu=\{E \cap D \mid E \in \delta\}$ on $D$ and let $H=\left\{\alpha \in Z^{D} \mid \alpha(B)=0\right.$ for some $\left.B \in \mu\right\}$. Then $G\left(R_{M}\right)=Z^{D} / H$.

In [4] an ideal $I$ of a ring $R$ is called local if it is contained in a unique maximal ideal. In $[\mathbf{2}, \mathbf{4}]$ the decompositions of ideals of $A(X)$ into local ideals were studied and in $[3,20]$ the primary ideals of $A(X)$ were studied where $X$ is a non-compact connected Riemann surface. We add a few remarks on these ideas. 
Proposition 2.1. Let $R$ be a Bezout domain such that each non-zero prime ideal of $R$ is contained in a unique maximal ideal. The following properties of a non-zero ideal $I$ of $R$ are equivalent.

(1) $I=I R_{M} \cap R$ for some maximal ideal $M$.

(2) I has prime radical.

(3) $I$ is a local ideal.

Proof. $(1) \Rightarrow(2)$. Since $R_{M}$ is a valuation ring $I R_{M}$ has prime radical, which we denote by $P$. Then $P \cap R$ is the radical of $I R_{M} \cap R$. (2) $\Rightarrow(3)$ is clear. (3) $\Rightarrow(1)$. This follows since for any ideal $I=\bigcap\left\{I R_{M} \cap R \mid M\right.$ is a maximal ideal of $\left.R\right\}$.

Now let $R$ be a Bezout ring as in the above proposition. Then for any ideal $I$ of $R, I=\bigcap\left\{I R_{M} \cap R \mid M\right.$ is a maximal ideal of $\left.R\right\}$ gives a decomposition of $I$ as an intersection of local ideals and these local ideals are irreducible by [13, Theorem 8]. For each maximal ideal $M$ of $R$ let $\mathscr{I}(M)=\left\{I \mid I\right.$ is an ideal such that $\left.I R_{M} \cap R=I\right\}$. Then the set of local ideals of $R$ is partitioned into the sets $\mathscr{}(M), M$ a maximal ideal of $R$, and for each maximal ideal $M, I \rightarrow I R_{M}$ gives a bijection between the elements of $\mathscr{I}(M)$ and the ideals of $R_{M}$. The primary ideals of $R$ are of course local ideals and this bijection preserves primary ideals. Thus the analysis of the local and primary ideals reduces to studying the ideal theory of $R_{M}$ for maximal ideals $M$. If further, $R$ is a $W$-ring with HT $(R)$ complete, then the study of the ideal theory of $R_{M}$ translates into an analysis of the value group of $R_{M}$ and this has been determined as an ultrapower $Z^{D} / H$. Thus locally the ideal theory of one such (non-Noetherian ) $W$-ring looks like the ideal theory of any other. We make this more precise in the next proposition.

Proposition 2.2. If $R$ and $S$ are non-Noetherian $W$-rings with $\mathrm{HT}(R)$ and $\mathrm{HT}(S)$ complete, and $V$ is any valuation overring of $R$, then there exists a valuation overring $V^{\prime}$ of $S$ with $G(V) \cong G\left(V^{\prime}\right)$, and hence $V$ and $V^{\prime}$ have isomorphic lattices of ideals.

Proof. Since any overring of a Bezout domain is a localization [7, Theorem 27.5] we have $V=R_{P}$ for some $P \in \operatorname{Spec}(R)$. Further, if we let $M$ be a maximal ideal of $R$ containing $P$ then $G\left(R_{P}\right)$ is a quotient of $G\left(R_{M}\right)$ by an isolated subgroup of $G\left(R_{M}\right)$. Thus it suffices to consider the case that $V=R_{M}, M$ a maximal ideal. If $M \in X(R)$ the result is trivial, so we may assume that $M$ is a free ideal. Let $D \in Z(M), D \neq X(R)$. Let $E \neq X(S)$ be an infinite permissible subset of $X(S)$, and let $\varphi: D \rightarrow E$ be a bijection. Then $\mu_{1}=\{D \cap H \mid H \in Z(M)\}$ is an ultrafilter on $D$ and so $\mu_{2}=\left\{\varphi(B) \mid B \in \mu_{1}\right\}$ is an ultrafilter on $E$. Let $\delta=\left\{H \in Z(S) \mid H \cap E \in \mu_{2}\right\}$. Then $\delta$ is a $\Delta$-ultrafilter on $X(S)$. Let $N=Z^{-1}(\delta)$ be the corresponding maximal ideal of $S$. Then $S_{N}$ is a valuation ring whose value group $G\left(S_{N}\right)$ is $Z^{E} / \mu_{2} \cong Z^{D} / \mu_{1}=G\left(R_{M}\right)$.

Corollary. Any two non-Noetherian W-rings $R$ with $\mathrm{HT}(R)$ complete have the same dimension.

3. W-rings as rings of analytic functions. Let $K$ be a field containing $\mathbb{C}$ as a subfield. A $\mathbb{C}$-rational place of $K$ is a place $s: K \rightarrow \mathbb{C} \cup\{\infty\}=\Sigma$ which maps $\mathbb{C}$ onto $\mathbb{C}$. Let $S$ be the 
set of $\mathbb{C}$-rational places of $K$. We get a natural map $\varphi: K \rightarrow \Sigma^{S}$ defined by $\varphi(f)(s)=s(f)$. It is well-known [6] that if $K$ is an algebraic function field in one variable over $\mathbb{C}$, then $S$ is in a natural way a compact connected Riemann surface such that $\varphi$ identifies $K$ with the set of meromorphic functions on $S$, and every compact connected Riemann surface is of this form. A similar result for open Riemann surfaces has remained an elusive problem $[14,19]$. It was shown by Iss'sa [12] that if $X$ is an open connected Riemann surface, then $X$ is uniquely determined as a Riemann surface by its field $M(X)$ of meromorphic functions. There remains the problem of determining those fields $F$ which are of the form $M(X)$, or equivalently those rings $R$ of the form $A(X)$, for some open Riemann surface $X$. In particular does it hold that every $W$-ring $R$ with coefficient field $\mathbb{C}$ and $H T(R)$ complete is of this form? Let $R$ be a $W$-ring with coefficient field $\mathbb{C}$ and $\mathrm{HT}(R)$ complete. Then each point $P \in X(R)$ defines a $\mathbb{C}$-rational place $s_{P}$ by $s_{P}(a)$ is the residue class of $a$ in $R / P=\mathbb{C}$ if $a \in R_{P}$, and $s_{P}(a)=\infty$ if $a \in K \backslash R_{P}$, where $K$ is the quotient field of $R$. We get a natural map $\varphi: K \rightarrow \Sigma^{X}$ where $\Sigma=\mathbb{C} \cup\{\infty\}$. If $X$ is uncountable, then since each $a \in R$ has at most countable many zeros, it follows that $\varphi$ is injective. If $R=A(Y)$ for some Riemann surface $Y$, then $Y$ would correspond to $X$ as a point set, and would have the weakest topology such that all of the elements of $\varphi(K)$ are continuous. Further, each $\mathbb{C}$-rational place of $K$ whose valuation ring is rank one discrete, would be of the form $s_{P}$ for some $P \in X$ [12]. Besides the given topology on a $W$-ring $R$, the embedding $\varphi: R \rightarrow \Sigma^{X}$ allows one to give $R$ the compact-open topology (which is in general weaker than the given topology). Call $R$ with this topology $A$. If $X$ is second countable and locally compact, then it can be seen that $A$ is also a $W$-ring with $\operatorname{HT}(A)$ complete, but it remains to determine a conformal structure on $X$. While we do not know what conditions on $R$ are required for $A$ to be $A(X)$ we note the further condition that for every $a \in A$ there must exist a ring homomorphism $f_{a}: A(\mathbb{C}) \rightarrow A$ such that $f_{a}(z)=a$ where $z: \mathbb{C} \rightarrow \mathbb{C}$ is the identity function. That is $A(\mathbb{C})$ is a free object on one generator in the category of rings of analytic functions on open Riemann surfaces. This implies that each $\mathbb{C}$-rational place of $K$ having rank one discrete valuation ring, is of the form $s_{P}$ for some $P \in X$.

\section{REFERENCES}

1. N. L. Alling, The valuation theory of meromorphic function fields over open Riemann surfaces, Acta Math. 110 (1963), 79-96.

2. N. L. Alling, The valuation theory of meromorphic function fields, Proc. Sympos. Pure Math. Vol. 11, Amer. Math. Soc., Providence, R.I., 1968, pp. 8-29.

3. N. L. Alling, Primary ideals in rings of analytic functions, Proc. Amer. Math. Soc. 53 (1975), 423-427.

4. N. L. Alling, Global ideal theory of meromorphic function fields, Trans. Amer. Math. Soc. 256 (1979) 241-266.

5. K. Aoyama, On the structure space of a direct product of rings, J. Sci. Hiroshima Univ. Ser. A-I, 34 (1970), 339-353.

6. C. Chevalley, Introduction to the theory of algebraic functions in one variable, Math. Surveys No. VI, Amer. Math. Soc. 1951.

7. R. Gilmer, Multiplicative ideal theory (Marcel Dekker, 1972). 
8. U. Güntzer, Zur Funtionentheorie einer Veränderlichen über einen vollständigen nicht archimedischen Grunkörper, Arch. Math. 17 (1966), 415-431.

9. O. Helmer, Divisibility properties of integral functions, Duke Math. J. 6 (1940), 345-356.

10. M. Henriksen, On the ideal structure of the ring of entire functions, Pacific J. Math. 2 (1952), 179-184.

11. M. Henriksen, On the prime ideals of the ring of entire functions, Pacific J. Math. 3 (1953), 711-720.

12. H. Iss'sa, On meromorphic function fields on a Stein variety, Ann. of Math. 83 (1966), $34-46$.

13. C. U. Jensen, Arithmetical rings, Acta Math. Acad. Sci. Hungaricae 17 (1966) 115-123.

14. S. Kakutani, Rings of analytic functions, in Lectures on functions of a complex variable, edited by W. Kaplan et al. (Univ. of Michigan, 1955), 71-83. (1975).

15. J. Klingen, Eine topolgische Verallgemeinerung von Krullringen, Dissertation Univ. Köln

16. J. Klingen, Uber eine topologische Verallgemeinerungen von Krullringen, Math. Nachr. 91 (1979), 59-76.

17. M. Krasner, Rapport sur le prolongement analytique dans les corps valués complets par la méthode des éléments analytiques quasi-connexes, Bull. Soc. Math. France Memoire 39-40 (1974), 131-254.

18. M. Lazard, Les zéros des fonctions analytiques d'une variable sur un corps valué complet., Institut des Hautes. Études Scientifiques 14 (1962), 223-251.

19. H. Röhrl, Unbounded coverings of Riemann surfaces and extensions of rings of meromorphic functions, Trans. Amer. Math. Soc. 107 (1963), 320-346.

20. R. Williams, Primary ideals in rings of analytic functions, Trans. Amer. Math. Soc., 177 (1973), 37-49.

\section{UNIVERSITY OF CALIFORNIA \\ RIVERSIDE CA 92521 \\ USA}

